\title{
Survey on Noise Removal in Digital Images
}

\author{
B.Mohd. Jabarullah ${ }^{1}$, Sandeep Saxena ${ }^{2}$, Dr. C. Nelson Kennedy Babu ${ }^{3}$ \\ ${ }^{1}$ (Research Scholar, Computer Science, M.S. University, India) \\ ${ }^{2}$ (Research Scholar,Computer Application, IFTM University, India) \\ ${ }^{3}$ (Principal, CMS College of Engg., India)
}

\begin{abstract}
When an Image is formed, factors such as lighting (spectra, source, and intensity) and camera characteristics (sensor response, lenses) effect the appearance of the image. So, the prime factor that reduces the quality of the image is Noise. Noise hides the important details of images. To enhance the image qualities, we have to remove noises from the images without loss of any image information. Noise removal is one of the pre-processing stages of image processing. There are different types of noises which corrupt the images. These noises are appeared on images in different ways: at the time of acquisition due to noisy sensors, due to faulty scanner or due to faulty digital camera, due to transmission channel errors, due to corrupted storage media. In order to get enhanced images, many researchers present several methods to remove noises from different types of images by preserving important details like structural features, textural information. In this paper, we present a survey on types of Noises, types of images and noise removal algorithms. We have considered three types of noises: Impulse noises, Speckle noise, Gaussian noise from two most useful images: sensor images, medical images and gray scale images. We analyze all noise removal algorithms for each noise from each of these images. At the end of our study, we present comparative study of all these algorithms and, conclude with several promising directions for future research work.
\end{abstract}

Keywords: Noise, Structural features, textural information, Impulse noise, Speckle noise, Gaussian noise, sensor images, medical images, gray scale images.

\section{Introduction}

Since last decades, researchers are involved on face recognition in image processing and they achieved so many mile stone for this. Because face recognition is the critical stage to identify the face in images due to pose, presence or absence of structural components, facial expression, occlusion, image orientation. Above all, Noise is prime factor of reducing face recognition rate. Several methods have been evolved to increase recognition rate. Ming-Hsuan Yang et al [1] classified these methods into four categories: Knowledge-Base Methods, Feature Invariant Approaches, Template Matching Methods, and Appearance -Based Methods. These methods will perform well when the test image is noise free. So, Noise plays vital role in image processing stage [2][3].

In order to identify faces in an image, images are enhanced by removing noise in the image with preserving important information of the image. These noises are generally affected the quality of the image and formed during images captured from the sources like sensor, digital camera, CCTV, stored in storage media [3]. There are two types of images: gray scale image and color image. Similarly noises are impulse noise, speckle noise, Gaussian noise, striped noise. Many researchers have achieved a mile stone to develop algorithms to remove/reduce noises. An attempt has made in this paper to study and review all the algorithms. As a result of this study, it suggests to find best algorithm for each noise removal from each images.

With an aim to find best suited noise removal algorithm for each type of image, the rest of this paper is organized as: Section II presents the detailed review of all noise removal algorithms. Section III presents comparative study. Section IV presents conclusion.

\section{Noise Removal Algorithms}

In this section, we review exiting Noise Removal algorithms to enhance the quality of images. We have considered three types of images according to applications of images, to device used to capture/store and to most widely used images: sensor images, medical images, gray scale images and most frequently occurred noises in these images: Impulse, Speckle and Gaussian noises. So, we classify noise removal algorithms into three types according to these three different noises and images as given below:

1) Filtering methods for impulse noise in sensor images

2) Filtering methods for speckle noise in medical images

3) Filtering methods for Gaussian noise in gray scale images 


\subsection{Filtering Methods for Impulse Noise in Sensor Images}

L. Ganesh et. al [4] presented image fusion algorithm for impulse noise removal. In general, images are captured by different sensors, camera or other capturing devices. Here, different sensors are taken into an account and these sensors are produced different impulse noisy images. Noisy image is individually filtered by various non linear filters such as Vector Median Filter (VMF), Rank Conditioned VMF, Rank Conditioned and Threshold VMF, Counter Weighted VMF, Absolute Deviation VMF. Now, Image fusion techniques are applied on this filtered image to fuse into single image. And then, absolute deviation VMF is again applied on this fused images to create filtered fused image which gives the best result than fused image. Noisy density of using image is range from $10 \%$ to $60 \%$. The performance of each filter is evaluated by performance metrics; Mean Square Error, Peak Signal to Noise Ratio, and Structural Similarity Index and the result suggested that filtered fused image gives the best result compared with all independent non linear filters.

J. Harikiran et al [5] also presented image fusing technique to remove impulse noise. In this paper, images affected by impulse noises i.e salt and pepper noise, random valued impulse noise are filtered by order statistics filters which gives better performance as compared to linear filters. Performance metrics of each order static filters i.e. Median Filters (MF), Vector Median Filter (VMF), Basic Vector Directional Filter (BVDF), Spatial Median Filter (SMF), Modified Spatial Median Filter (MSMF) individually compared with Fused Image. Canny filter is also used in the fused image to detect edges for further processing

Instead of using non linear filters and fusing technique in [5], an attempt has made to apply Fuzzy Filters for impulse noise reduction in [6]. The fuzzy filters are Fuzzy Weighted Fuzzy mean filter (FWM), Fuzzy Median Filter (FM), Weighted Fuzzy Mean Filter (WFM). The first Adaptive Weighted Fuzzy Mean filter (AWFM1), the second Adaptive Weighted Fuzzy Mean filter (AWFM2), the Fuzzy Decision Directed filter (FDD), Fuzzy Inference Ruled by Else action (FIRE) filter. All these filters are considered for all types of impulse noise reduction. These methods are compared with classical filters both linear and non linear filter methods (such as Adaptive Weighted Mean filter, Standard Median filter, Adaptive Wiener filter, Gaussian filter). Images are corrupted by impulse noises with different density 3\%, 15\% and 30\%. Density level is mainly classified into two categories: Low and high density. For low noise level, DS-FIRE, PWL-FIRE of fuzzy filters work well and for high noise level AWFM2 works well. This is also compared with visual point of view in addition to numerical point of classification.

Rong Zhu et al [7] developed improved algorithm of Median filter to remove salt and pepper noise of image. This improved Median Filter algorithm detects image noise, and establishes noise marked matrix according to characteristics of noise. Here, tt does not process the pixel marked as signal. Since, the Median filter is the best and used widely because of its capability of noise removal and high computational efficiency. But the function of Median filter is uniformly replaces the gray value of every pixel by the median of its neighbors. When the noise level is high, it removes important details of image. In order to preserve fine details of the image, the author developed improved algorithm of median filter based on local histogram. The histogram is constructed to find impulse noise pixels. For each possible gray scale value of pixels, histogram shows the amount of noise detections in the image. High peak value of histogram concludes the presence of impulse noise. The performance of improved median filters is tested with different noise density varied from $10 \%$ to $50 \%$ with increment of $10 \%$. It revealed that the proposed method gives better noise removal based on performance metrics. The experimental result suggested that it maintains the image details better and it is also more suitable for ordinary computer image de-noising.

The most commonly used method to remove impulse noise is median filter. But it does not preserve edges of the image. The challenging task is to preserve the edges of the image, several methods have been proposed. A new method was developed to remove impulse noise with preserving edges of the original image. J. Aalavandan et. al [8] proposed Adaptive Switching Median Filter (ASMF). This method is modified method of Switching Median Filter (SMF). Noise removal of this method is done by two stages. In the first stage, identifying noisy region. Here, a binary image is created using thresholding values where 0 's in the binary image defines noise free pixel and is defines noisy pixels. In the second stage, using Adaptive Switching Median Filter, removal of noise. According to performance metrics, this proposed method gives best performance while preserving significant and edge details. This proposed method is capable of removing Salt and Pepper noise.

K.Ratna Babu et. Al. [9] proposed a method Modified Median Filter to remove salt and pepper noise. In this proposed method, dummy rows and columns are added at each border to preserve edges. $3 \times 3$ neighborhood window is considered and central pixel of the neighborhood window is the processing pixel. A vector is used to maintain all neighborhood pixel intensity values except processing pixel. If all intensity values in the vector is 0 or 255 (Noisy), then processing pixel is replaced by mean value of all vector values. Here, instead of median value, mean value is calculated. If the intensity value of this vector is other than 0 or 255 , then processing pixel value is replaced by the median of values in the vector. This proposed method performs well up to $80 \%$ of noise density. 
M. Sreedevi et. al [10] proposed a method to remove impulse noise using Min-Max filter and MidPoint filter. The min-max filter is applied to find darkest points in the image and to reduce salt noise, and to find brightest points, to reduce pepper noise in the image. Midpoint algorithm is used to compute midpoint to compute midpoint between minimum and maximum values. This process is for all corrupted pixels in the image. This proposed method yields better result for the noise density level up to $70 \%$.

Instead of classical or improved method to remove impulse noise, another dimension of research is a decision based method has been adopted. Mahantesh R Choudhari et al [11] presented comparative study of all modern denoising algorithms and proposed a decision based method to remove salt and pepper noise. They considered Level-I and Level-II Improved Tolerance based Selective Arithmetic Mean Filtering (ITSAMFT) Technique, Tolerance based Selective Arithmetic Mean Filtering Technique (TSAMFT), and Median Filtering Algorithm. The most popular method to remove salt and pepper noise is median filtering methods, and its specialized median filtering methods. When the noise density level is high, then this method gives blurring of the original image and affects some fine details of the image. As the result suggest, based on performance metrics that ITSAMFT works well even the image has high density noise and this method preserves all details of the image and edges of the image. In the TSAMFT method, Arithmetic Mean Value is calculated based nonnoisy pixels or all the pixels in the mask of $\mathrm{m} x \mathrm{n}$ size. Tolerance is used to define intensity replacement of interested pixel (central pixel of the mean mask). In the improved method, Arithmetic mean value is calculated based on pixels specified range or pixels specified out of range value in the extended mask size 5 x 5 . Level-II ITSAMFT for the noise density 95\% gives better performance than the Level-I ITSAMFT, TSAMFT \& Median Filter, and this improved method is being consistently effective in noise suppression and detail preservation for various images.

R. Pushpavalli et al [12] presented a new method for image enhancement. The proposed Switching Median Filter technique is more effective in eliminating impulse noise. In this proposed method, if the processing pixel intensity lies between minimum and maximum pixel value of the image in the selected mask then this processing pixel is uncorrupted and remains as it is. If does not lie, then this corrupted processing pixel intensity is changed by median pixel value or already processed immediate neighboring pixel. This switching Median Filter is achieved well in eliminating impulse noise up to $70 \%$ of noise density with preserving edges and fine details.

\subsection{Filtering Methods For Speckle Noise in Medical image}

Y.Murali Mohan Babu et. al. [13] proposed a naval Bayesian based algorithm with in the frame work of wavelet analysis. In this paper, author has taken different wavelet techniques like Haar wavelet, Db4 wavelet, Sym wavelet, and bior wavelet with various thresholding techniques such as soft thresholding, hard thresholding, Bayes Soft thresholding. In this study, wavelet with Bayes soft thresholding gives the best performance and preserve features of the image.

Rakesh Singh et al [14] presented a comparative Analysis of Speckle Noise reduction techniques and their affect on image edge localization. Speckle noise is the multiplicative in nature. It degrades quality of the image by affecting bright areas of an image and not in the dark areas. Speckle noise removal is more difficult to remove as it is multiplicative form than the additive form of noises. In this paper, the two categories of speckle noise reduction are compared. The first category is anisotropic diffusion method based non linear Partial Differential Equation (PDE) and second one is wavelet based method using decimated and un-decimated wavelet. In this paper, the proposed method is to the input image decompose in to fine detailed coefficient using decimated or the un-decimated wavelets and appropriate wavelet family orthogonal or bi-orthogonal. Soft threshold is applied to suppress the noise. Inverse wavelet transform is used to get enhanced image. Analyzed result shows wavelet based method gives best result. Among all wavelet based methods, un-decimated orthogonal wavelet gives best result.

Soft thresholding using wavelet is presented in the above method. Manish Goyal et al [15] is proposed a method hybrid threshold technique using wavelet for speckle noise reduction. Here, corrupted image is decomposed to obtain sub bands using Discrete Wavelet Transform 2-level image decomposition. Threshold values for each sub-band coefficient are obtained using soft threshold technique and finally wiener filter method is applied. As the result suggested, this proposed method works well with different noise levels of different standard deviations. The experimental results are analyzed both qualitatively and quantitatively.

T. Sreekanth Rao et. al [16] proposed Wavelet Based Image de-noising of Non- logarithmic transformed data. Speckle noise is multiplicative in nature. So, It is converted in to additive noise by taking logarithm, in the method introduced above. But, here, the author attempt image de-noising by without taking logarithmic transformation and applied single level decomposition. Speckle corrupted image is decomposed to obtain sub bands. The standard deviation of each block of all sub bands is compared to the variation factor of corresponding block in high-high sub band, If $\sigma$ (standard deviation) is less than $\zeta$ (variation factor), each pixel in that block is replaced by its pixel mean value. Otherwise, the block is kept unchanged. Discrete Wavelet 
Transform (DWT) and Double Density-Discrete Wavelet Transform (DD-DWT) is applied for wavelet decomposition. Inverse Wavelet Transform finally applied on sub-bands to obtain de-noised image. The proposed method is analyzed and compared with other de-noising techniques like Soft Thresholding, Hard Thresholding and Lee filter. This proposed method shows minimum Mean Square Error.

R. Sivakumar et. al [17] has made an attempt in comparative study of speckle noise reduction in ultrasound B-scan images. This paper addressed the wiener filtering in wavelet domain with soft thresholding as the best method when compared with classical speckle noise reduction technique. The noisy input image is decomposed in to subbands. In order to remove noise in each sub band, wiener filter is used with soft thresholding and using inverse Dicrete Wavelet transform, image is reconstructed from the denoised sub bands. This proposed method revealed that this method produces the best result based on visual quality and performance metrics.

\subsection{Filtering Methods For Gaussian noise in Gray Scale images}

V.R. Vijay Kumar et. al [18] presented adaptive window based efficient algorithm for removing Gaussian noise in gray sale and color images. In this method, noise variance is calculated in the flat region of the corrupted image to define threshold value. Now an adaptive window of size $3 \times 3$ is formed. If the variance of the window is less than threshold, mean value of the window replaces the processing pixel of the window. If not, size of the window is to increase. This method is performs effectively for low to high density noise present in images.

M.S Safari et. al [19] is presented FIR filter based Genetic mixed noise removal. A window of size $5 \mathrm{x}$ 5 is considered. If the window is located in no abrupt changes in gray levels i.e. flat area, estimation of central pixel is the average of all the pixels in window surrounding the central pixel. If it is not flat area, abrupt changes in intensity of the pixel, estimation of central pixel is the average of only similar pixel surrounding the central pixels. In order to avoid conversion between real valued and bit string, real valued chromosomes are used instead of bit strings. Experimental result reveled the proposed filter has shown better performance than the wiener and median filter, as the case of variance in the salt and pepper noise density from 0 to 0.4 , but not in the case of variance in the Gaussian noise.

Tuan-Anh Nguyan et. al [20] presented spatially adaptive de noising algorithm for a single image corrupted by Gaussian noise. In this work, local statistics such as local weighted mean, local weighted activity, and local maxima are used to detect the noise. In order to suppress the additive noise, a spatially additive Gaussian filter is used. Because this filter is an adequate way to handle the degree of local smoothness since it is represented as function of local statistics. In this proposed method, the parameters like computational cost, oversmoothness, detection error, smoothing degree of re-constructed image are taken in to an account to effectively remove the noise components.

Yiwen Qiu et. al [21] presented as adaptive image de-noising method for mixture Gaussian noise in an image. The improved version of Immerkaer at [22] is developed a method for noise estimation. It combines block based method with filter based method to yield noise standard deviation (stable estimate deviation) with a low computational load. With the help of standard deviation obtained in the noise estimation stage, adaptive denoising method is used for noise removal. As a result suggested that this proposed method works well based on performance metrics.

Rashi Agarwal et. al [23] proposed a bit plane average filtering to remove Gaussian noise. The modified average filter is adopted. The noise corrupted image is sliced at different bit planes and on each bit planes, moving average filtering is applied. After that, arrange all bit-planes in their order of importance to recreate the image. In this paper, performance metrics for Moving Averaging Filter (MAF) and Bit-Plane Moving Average Filtering (BPMAF) have shown that the Bit-Plane Moving Average Filtering method yields very good result as compared with any other method including MAF.

Vishal Gard et. at [24] presented image denoising using curvelet transform using Log Gabor filter. Instead of using low pass filtering, curvelet transform is compared with Gabor filter. Apart from normal curvelet transformation, the given image is divided in to resolution layers; each layer contains details of different frequencies. These frequencies are attenuates and approximate with the help of Log Gabor filter. The performance metrics is used to measure quantitatively. This proposed method is compared with normal curvelet. As per experimental result, curvelet with Gabor filter is very effective in removing Gaussian noise and perform better than curvelet transform without Gabor.

Kun He et.al [25] proposed an algorithm based on the local feature of the image to eliminate Gaussian noise. The binary morphology is used to extract the edge and the texture of the image and then locate the noise points. Mean value of the non-noise points in the adaptive neighborhood is applied to eliminate noise for noise pixels which are not on the edge or the texture. Suppose, if the noisy pixels are on the edge or texture region, smoothened these region by using the pixels points of the neighborhood edge and texture. If noisy pixels/points are located on the smooth region, then noises are eliminated by using adaptive neighborhood. This method 
works well for smooth region of the image and adopts local smooth for edge and texture region. So, the image still has residual noise and it is mainly located on the image edge and texture.

Tuan- Anh Nguyan et. al [26] presented fast and efficient Gaussian noise image restoration algorithm by spatially adaptive filtering method. This work is similar to presented at [21]. In this method also, noise removal using local statistics has noise detection and these noise removal stages with the help of local statistics and modified Gaussian filter algorithm, effective noise suppression is achieved.

\section{Performance Evaluation}

The reported performances of three main categories of noise removal algorithm described in the previous section are analyzed. Table I summarizes the performance of different filtering methods for impulse noise and it mostly affects the sensor images. The main objective of noise removal is to image enhancement and better recognitions rate. The Mean Square Error (MSE) and Peak Signal Noise Ratio (PSNR) are used to evaluate quality of the image after applying noise removal methods.

TABLE I Performance of Filtering Methods for Impulse Noise

\begin{tabular}{|c|c|c|c|c|}
\hline S.No & Filtering Method & Noise Density & MSE & PSNR \\
\hline 1 & Fused Filter Image [4] & $60 \%$ & 125 & 27.19 \\
\hline 2 & Fusing Technique [5] & $40 \%$ & 10.63 & 37.87 \\
\hline 3 & Fuzzy Filters [6] & $30 \%$ & 89.46 & 28.61 \\
\hline 4 & $\begin{array}{c}\text { Improved Median Filter based on } \\
\text { local list [7] }\end{array}$ & $50 \%$ & 28.4 & 33.59 \\
\hline 5 & $\begin{array}{c}\text { Enhanced Switching Median } \\
\text { Filter [8] }\end{array}$ & $65 \%$ & 4.74 & 41.37 \\
\hline 6 & Modified Median Filter [9] & $80 \%$ & 228.4549 & 24.54 \\
\hline 7 & $\begin{array}{c}\text { Min-Max and Mid Point Filter } \\
\text { [10] }\end{array}$ & $70 \%$ & 162.53 & 26.02 \\
\hline 8 & Second Level of ITSAMFT [11] & $95 \%$ & 1028.13 & 18.01 \\
\hline 9 & Switching Median Filter [12] & $90 \%$ & 870.86 & 18.72 \\
\hline
\end{tabular}

In table I, the performance of various filter for the first category has considered i.e. filtering methods for impulse noise. Each method, in table I, is tested and compared with other methods. Here, the test images are collected the captured images by different sensors. Table I shows the method which works well for the particular density. Schneier M et. al [27] is proved, PSNR is in the range of 30-45 which means the nose free image after noise removal is similar to original image. So, above all methods are lying the range are produced good quality image. But Level 2 ITSAMFT has given excellent performance even if the noise density is too high. Similarly, switching median filtering technique is comparatively good for high density noise. It is also observed that when MSE decreases, then PSNR increases. Minimum MSE is obtained in the ESMF ( at Sr. No. 5) and PSNR is also in the range 30-45. But it works well up to 65\% of noise density. The result prevails that all filtering methods work well up to $60 \%$ of average noise density. If it increases, quality gets affected.

Table II Performance of Filtering Methods for Speckle Noise

\begin{tabular}{|c|c|c|c|c|}
\hline Sr No & Filtering Method & $\begin{array}{c}\text { Noise } \\
\text { Density }\end{array}$ & MSE & PSNR \\
\hline 1 & $\begin{array}{c}\text { Bayesian-based algorithm in wavelet } \\
\text { Transform [13] }\end{array}$ & 0.1 & 0.094 & 58.39 \\
\hline 2 & $\begin{array}{c}\text { Wavelet Transform with soft } \\
\text { thresholding: Un decimated Orthogonal } \\
\text { Wavelet [14] }\end{array}$ & 0.6 & 14.32 & 36.57 \\
\hline 3 & $\begin{array}{c}\text { Hybrid Techniques based on Wavelet } \\
\text { Thresholding [15] }\end{array}$ & 0.09 & 98.22 & 28.26 \\
\hline 4 & Orthogonal Wavelet Transform [16] & 0.8 & 17.36 & 35.78 \\
\hline 5 & $\begin{array}{c}\text { Wiener filtering with Bayes Shrink } \\
\text { Thresholding [17] }\end{array}$ & 0.06 & 65.93 & 29.93 \\
\hline
\end{tabular}

The second category of the classification i.e. filtering methods for Speckle noise has taken for the discussion and performance of various methods shown in Table II. Bayesian based algorithm in wavelet transform gives best result when it is compared with different wavelet techniques. Noise density level is above $50 \%$ in the un-decimated orthogonal wavelet but less compare with Bayesian model. It produces better quality of the image and it preserves edges of the image also. The noise level has taken for the speckle noise removal 
from 0.01 to 0.09 . This high density noise level is close to Bayesian model. The method applied to suppress instead removal is hybrid Technique on wavelet thresholding. This method is also best in edge preservation when compare with traditional techniques. In the Orthogonal wavelet thresholding method, heavily corrupted by noise is considered and this performed well compared with all the other methods in Table II. The performance study of image will play important role based on number of images are used. In this concept, wiener filtering with Bayshrink thresholding method tested 200 images. In perceptional view of all the methods given in Table II, wavelet based technique gives much improved result, provide high degree of performance.

The third classification of this survey is based on performance of filtering methods for Gaussian noise removal, shown in Table III. In general, the Gaussian noise occurs during image storing, capturing by counting photons. Gaussian noise affects each pixel in the image by a small amount based on standard deviation, in [28]. One of the methods to remove the Gaussian noise is Adaptive window method. This method is applied for removal of Gaussian noise in gray scale image and color image. In every noise corrupted image, it is very difficult to distinguish noise and edge information. So, in order to preserve edges, fuzzy concept is used. The

Table III Performance of Filtering Methods of Gaussian Noise

\begin{tabular}{|c|c|c|c|c|}
\hline Sr No & Filtering Method & $\begin{array}{c}\text { Noise } \\
\text { Density }\end{array}$ & MSE & PSNR \\
\hline 1 & $\begin{array}{c}\text { Adaptive window based efficient } \\
\text { Algorithm [18] }\end{array}$ & 30 & 9.02 & 27.12 \\
\hline 2 & $\begin{array}{c}\text { FIR-Filter based Fuzzy Genetic Mixed } \\
\text { Noise removal [19] }\end{array}$ & 5 & 2542.9 & 14.07 \\
\hline 3 & $\begin{array}{c}\text { Spatially adaptive de-noising algorithm } \\
{[20]}\end{array}$ & 30 & 31.9 & 33.09 \\
\hline 4 & $\begin{array}{c}\text { An Adaptive Image De-noising Method } \\
{[21]}\end{array}$ & 10.84 & 14.178 & 36.61 \\
\hline 5 & Bit Plane Average Filtering [23] & 20 & 96.63 & 28.28 \\
\hline 6 & Curvelet Transform with Gabor filter \\
{$[24]$} & 3 & .02442 & 64.25 \\
\hline 7 & Local Feature Method [25] & 40 & 632.89 & 20.11 \\
\hline
\end{tabular}

image may be corrupted by mixed noises and it's a challenging task to identify noise type, affected area of the image, finally removal of the mixed noises. Image corrupted by Gaussian noise and Salt \& Pepper nose is considered and FIR Fuzzy filter is applied to remove these mixed noises. The performance of this method, as per experimental result, is decreased when the noise level is high. So, this is not suited for Gaussian noise removal. It is so important to reduce computational cost, blurring, and over smoothness as edge preservation. Keeping in view of these parameters, Spatial Adaptive method is best suited with high level of noise detection fidelity. The different regions in the images will be corrupted by different noises. An Adaptive image de-noising method is applied and tested on such images. One region in the image is affected low density noise and other region in the image is affected by high density noise. Out come of spatially adaptive de-noising method is higher than the adaptive image de-noising method. But this out come is almost similar to Bit Plane average filtering method. High contrast image plays very important role in noise removal, emphasized in this method.

\section{Conclusion}

This paper attempts to present a comprehensive survey of research on noise removal methods. We have focused on only most frequently affected noises: Impulse Noise, Speckle Noise, and Gaussian Noise with various noise intensity range from low to high. We have analyzed noise removal algorithms for these noises. The parameters for this analysis were low cost, less time, high level of noise detection, preserving features and edges, over smoothness, high contrast image, high density noise, and mixture of noises. There is lack of uniformity in how methods are evaluated so it is imprudent to declare which methods indeed have lowest error rate with highest noise ratio. Therefore, our analysis has produced relative performance of methods. Our study has suggested relatively the best noise removal method in each noise, not in over all i.e applicable to Impulse noise, Speckle noise and Gaussian noise.

Therefore, noise removal is still a challenging task in order to obtain better recognition rate. So, keeping in view, a robust system should fulfill all the above parameters with multiple noises removal in a single image and in multiple images. 


\section{References}

[1] Ming-Hsuan Yang, David J. Kriegman, Narendra Ahuja," Detecting Faces in Images: A Survey",IEEE Transaction on Pattern Analysis and Machine Intelligence, Vol 24, No. 1, Jan 2002, pp 34-58

[2] Sarawat Anam, Md Shohidul Islam, M.A. Kasheem, M.N. Islam, M.R. Islam,”Face Recognition using Genetic Algorithm and Back Propagation Neural Network", Proceedings of the International Multi Conference of Engineers and Computer Scientists, March 1820, 2009

[3] S. Zeenathunisa, A.Jaya, M.A. Rabbani," A Biometric Approach Towards Recognizing Face in Various Dark Illuminations”, IEEE, 2011, pp 1-7

[4] L.Ganesh, S P K Chaitanya, J D Rao, M N V S S Kumar, "Development of Image Fusion Algorithm for Impulse Noise Removal in Digital Images using the Quality Assessment in Spatial domain", IJERA, Vol. 1, Issue 3, pp 786-792

[5] J. Harikiran, B. Saichandana, B. Divakar," Impulse Noise Removal in Digital Images”, IJCA, Vol. 10, No. 8, Nov 2010, pp 39-42

[6] Jyoti Chauhan,"A Comparative study of classical and Fuzzy Filters for Impulse noise reduction”, IJARCS, Vol. 3, No. 1, Jan-Feb 2012, pp 416-419

[7] Rong Zhu, Yong Wang," Application of Improved Median Filter on Image Processing”, Journal of Computers, Vol. 7, No. 4, April 2012, pp 838-841

[8] J. Aalavandan, Lt. Dr. S. Santhosh Baboo,” Enhanced Switching Median Filter for De-noising Ultrasound”, IJARCE, Vol. 3, No. 2, March-April 2012, pp 363-367

[9] K. Ratna Babu, L. Arun Rahul, P. Vineet Souri, A. Suneetha," Image Denoising in the presence of high level Salt and Pepper noise using Modified Median filter", IJCST, Vol. 2, Sp 1, Dec 2011, pp 180-183

[10] M. Sreedevi, G. Vijay Kumar, N.V.S. Pavan Kumar, “ Removing Impulse noise in gray scale images using Min Max and Mid Point Filters", IJARCS, Vol. 2, No. 6, Nov-Dec 2011, pp 377-379

[11] Mahantesh, R. Choudhari, Prof. K. Chandrasekar, Dr. S. A. Hariprasad," Comparison of Modern Denoising Algorithms", IJARCET,Vol. 1, Issue 4, June 2012, pp 388-394

[12] R. Pushpavalli, G. Sivaradje,’Switching Median Filter for Image Enhancement”, IJSER, Vol. 3, Issue 2, Feb 2012, pp 1-5

[13] Y. Murali, Mohan Babu, Dr. M.V. Subramanyam, Dr. M. N. Giri Prasad," Bayesian Denoising of SAR image”, IJCST, Vol. 2, Issue 1, Mar 2011, pp 72-74

[14] Rakesh Singh, Amandeep Kaur, "Comparative Analysis of Speckle Noise Reduction Techniques and their affect on Image Edge Locaization", IJCST, Vol.2 Issue 4, Oct-Dec 2011,pp 78-82

[15] Manish Goyal, Gianetan Singh Sekhon, “ Hybrid Threshold Technique for Speckle Noise Reduction using Wavelets for gray scale images", IJCST, Vol. 2, Issue 2. June 2011, pp 620-625

[16] T. Sreekanth Rao, P. Gangamohan, P. Nagarjuna Reddy, B. Prathyusha," Wavelet Based Image De-noising of Non-Logarithmic Transformed Data", IJCST, Vol. 2, SP I, Dec 2011, pp 213-215

[17] R. Sivakumar, D. Nedumaram," Comparative Study of Speckle noise Reduction of ultrasound B-Scan images in Matrix Laboratory environment", IJCA, Vol. 10, No. 9, Nov 2010, pp 46-50

[18] V.R. Vijay Kumar, P.T. Vanathi, P. Kanagasabapathy," Adaptive Window Based efficient Algorithm for removing Gaussian noise in gray scale and color images", International conference on Computational and Multimedia Application”, IEEE Computer Society, 2007, pp 319-323

[19] M.S. Safari, A. Aghagolzadeh,” FIR filter based Fuzzy-Genetic Mixed noise removal”, IEEE 2007

[20] Tuan-Anh Nguyen, Won-Seon Song, Min-Cheol Hong," Spatially Adaptive Denoising algorithm for a single image corrupted by Gaussian noise", IEEE Transaction on Consumer Electronics, Vol. 56, No. 3, Aug 2010, pp 1610-1615

[21] Yiwen Qin, Zongliang Gan, Yaqiong Fan, Xiuchang Zhu, "An Adaptive Image Denoising method for Mixure Gaussian Noise", 2011, IEEE

[22] J. Immerkaer, "Fast Noise Variance Estimation", Computer Vision and Image Understanding- Academic Press, Vol 64, No 2, Sep 1996, pp 300-302

[23] Rashi Agarwal,’Bit Plane Average Filtering to remove Gaussian noise from High Contrast Image", International Conference on Computer Communication and Informatics (ICCCI-2012), Jan 10-12,2012, IEEE

[24] Vishal Garg, Nisha Raheja," Image Denoising using Curvelet Transform Using Log Gabor Filter", IJARCET, Vol. 1, Issue 4, June 2012, pp 671-679

[25] Kun He, Xin-Cheng Luan, Chun-Hua Li, Ran Liu," Gaussian Noise Removal of Image on the Local Feature”, IEEE Computer Society, 2nd International Symposium on Intelligent Information Technology Application, 2008, IEEE, pp 867-871

[26] Tuan-Anh Nguyen, Myoung-Jin Kim and Min-Cheol Hong," Fast and Efficient Gaussian Image Restoration Algorithm by Spatially Adaptive Filtering", 28th Picture Coding Symposium(PCS 2010), Dec 8-10, 2010, IEEE, pp 122-125

[27] Schneier. M and Abdel-Mottaleb. M ,'Exploiting the JPEG Compression Scheme for Image Retrieval”, IEEE Transaction Pattern Analysis and Machine Intelligence", Vol 18, No. 8, 1996, pp 849-853

[28] Rafael C. Gonzalez, Richard E. Woods, "Digital Image Processing - 3rd Edition", Pearson Education, Inc, publishing as Prentice Hall , 2008 